\section{Treatment of Dieulafoy's lesion of the right colon with epinephrine injection and argon plasma coagulation}

Dieulafoy's lesion is a tiny submucosal defect overlying an artery in the muscularis mucosa [1]. Dieulafoy's lesion of the colon is a rare cause of lower gastrointestinal bleeding [2].

A 63-year-old woman with multiple myeloma underwent autologous bone marrow transplantation and after 6 weeks developed massive hematochezia with hemodynamic instability. Colonoscopy demonstrated bright red blood in the terminal ileum, all of the colon, and the rectum ( $\bullet$ Fig. 1 ).

After the area had been washed with water, a point of spurting active bleeding was located in the ascending colon. We injected epinephrine and the bleeding stopped; identification of a minute mucosal defect was then possible ( $\bullet$ Fig. 2 ).

We complemented the treatment with argon plasma coagulation (APC) using a 2.3-mm probe, with flow rate of $1.0 \mathrm{~L} /$ minute and a setting of $40 \mathrm{~W}$ in order to minimize the risk of bowel perforation, until the lesion was completely coagulated. Submucosal injection of epinephrine has a protective effect when using thermal techniques in the right colon [3]. There was no rebleeding during the follow-up of 14 days. The patient died after this period from septic shock.

Dieulafoy's lesion in the setting of hemorrhagic shock has a high risk of rebleeding, justifying the addition of a complementary endoscopic treatment (thermal or mechanical) following epinephrine injection [4-5]. However, we have to keep in mind that the right colon has a thinner wall compared with the stomach, and use of band ligation and heat probe with high temperatures can lead to bowel perforation.

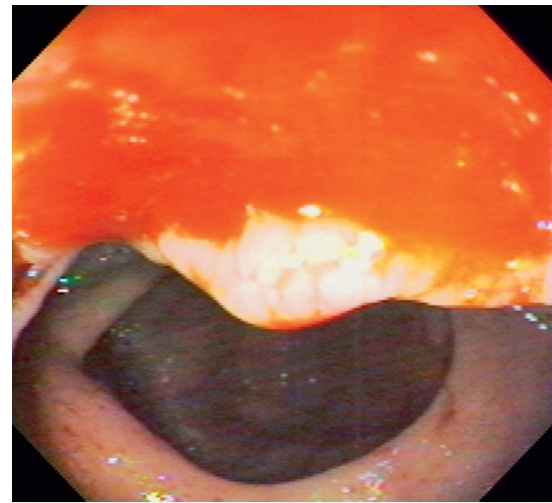

Fig. 1 Colonoscopy showing spurting active bleeding in ascending colon.

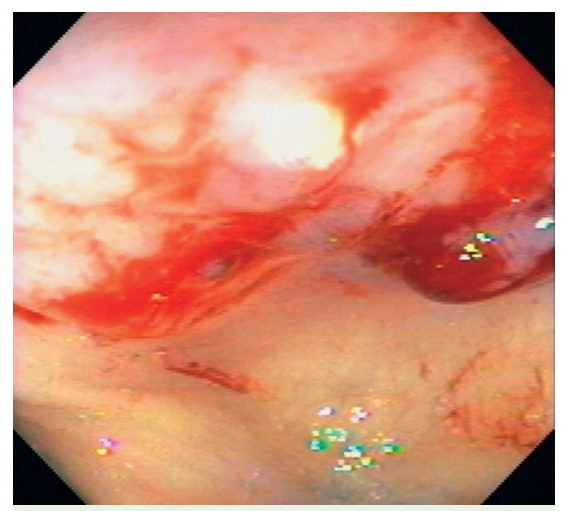

Fig. 2 Minute mucosal defect in ascending colon identified after injection of epinephrine.

To our knowledge, this is the first report of a combined endoscopic approach with injection of epinephrine and APC to treat a Dieulafoy's lesion of the right colon in a patient with significant thrombocytopenia. It seems to be a secure and effective modality of endoscopic therapy of bleeding in this setting, with minimum risk of perforation and high possibility of hemostasis.

Endoscopy_UCTN_Code_CPL_1AH_2AB

\section{J. L. S. Souza}

Department of Gastroenterology, Diagnostic Center in Gastroenterology, University of Sao Paulo, Brazil

\section{References}

1 Juler GL, Labitzke HG, Lamb R et al. The pathogenesis of Dieulafoy's gastric erosion. Am J Gastroenterol 1984; 79: 195-200

2 Norton ID, Petersen BT, Sorbi D et al. Management and long term prognosis of Dieulafoy lesion. Gastrointest Endosc 1999; 50: $762-767$

3 Suzuki N, Arebi N, Saunders BP. A novel method of treating colonic angiodysplasia. Gastrointest Endosc 2006; 64: 424-427

4 Calvet $X$, Vergara $M$, Brullet $E$ et al. Addition of a second endoscopic treatment following epinephrine injection improves outcome in high risk bleeding ulcers. Gastroenterology 2004; 126: 441 - 450

5 Chung IK, Kim EJ, Lee MS et al. Bleeding Dieulafoy's lesions and the choice of endoscopic method: comparing the hemostatic efficacy of mechanical and injection methods. Gastrointest Endosc 2000; 52: 721 724

Bibliography

DOI $10.1055 / \mathrm{s}-0029-1214774$

Endoscopy 2009; 41: E192

(c) Georg Thieme Verlag KG Stuttgart · New York . ISSN 0013-726X

Corresponding author

J. L. S. de Souza, MD

Department of Gastroenterology,

Diagnostic Center in Gastroenterology,

University of São Paulo

255 Dr Enéas de Carvalho Aguiar Ave.

9th floor - Room 9159

São Paulo

Brazil

Fax: +55-11-30697940

jlsebba@gmail.com 